\title{
Does laparoscopic surgery spell the end of the open surgeon?
}

\author{
Riaz Agha BSc Gordon Muir FRCS ${ }^{1}$
}

J R Soc Med 2003;96:544-546

Barely fifteen years have passed since the laparoscopic era was launched with a cholecystectomy by the French surgeon Philippe Mouret. ${ }^{1}$ Yet today by far the majority of cholecystectomies are done in this way, ${ }^{2}$ and laparoscopic surgery finds many other indications. The UK has been slower than some other countries to adopt these techniques; indeed, the President of the Association of Coloproctology, Neil Mortensen, has declared '. . . it is scandalous how far we are behind on keyhole surgery for bowel cancer and other bowel problems' (Daily Telegraph, 6 May 2003).

The range of operations now extends from simple procedures such as herniorrhaphy and ovarian cystectomy to complex operations including radical prostatectomy, nephrectomy, and adrenalectomy. ${ }^{3}$ Where will this end? Do such techniques spell the demise of open surgery? In discussing this matter we confine ourselves to laparoscopy, though the argument applies also to numerous other procedures whereby open operation is replaced by use of small incisions and long thin instruments, with display of the surgical field on a monitor.

The question in our title requires analysis of two key elements: is laparoscopic surgery better for the patient (without unduly hampering the surgeon); and is it practically and financially feasible?

\section{ADVANTAGES FOR THE PATIENT}

In patient terms, laparoscopic surgery has the advantages of avoiding large open wounds or incisions and thus of decreasing blood loss, pain and discomfort. Patients have fewer unwanted effects from analgesia because less analgesia is required. The fine instruments are less apt to cause tissue trauma and blood loss. The rate of postoperative complications is generally lower, especially those related to the wound such as dehiscence, infection, cellulitis and incisional hernia. ${ }^{4}$ Performance of the operation within the body cavity avoids the cooling, drying, excessive handling and retraction of internal organs associated with conventional 'open' techniques-possibly reducing postoperative

Guy's, King's and St Thomas' School of Medicine, London; ' ${ }^{1}$ Department of Urology, King's College Hospital, London SE5 9RS, UK peritoneal adhesions with their hazard of later bowel obstruction.

These benefits help to decrease the recovery period, thus lessening the risks of bone loss, muscle atrophy and urinary retention associated with lengthy bed rest and inactivity. Other benefits of early mobilization are lower rates of chest infection and deep vein thrombosis. Finally, patients prefer small scars to large ones, and laparoscopic surgery is likely to generate less postoperative anxiety related to self-image.

\section{PRACTICAL ASPECTS}

Laparoscopic surgery means less direct contact between the surgeon and the patient - and consequently less risk that the surgeon will acquire a virus infection from the patient or vice versa. Some surgeons, however, are worried that this distancing adds a layer of 'separation' that hampers surgical judgment. Moreover, there are other perceptual difficulties. After the trocar is inserted, the trocar site serves as both a fulcrum and a steadying point. A small movement at the proximal end gives a large movement at the distal end. The normal axis is inverted, in that to go left the trocar must be moved right and to go down it must be moved up; to turn it in circles one goes in the usual direction, but the instrument is $180^{\circ}$ from where one might suppose. The surgeon is effectively operating in a mirror.

The loss of tactile clues can be disadvantageous. Images from three-dimensional structures are transmitted via the laparoscope onto a two-dimensional screen, making it difficult to judge depth and reducing the perceptual cues for identification of anatomical structures. An added difficulty is that the visual field is smaller than with open surgery, and the necessity to work with screen images demands special mental as well as physical skills. ${ }^{5}$ (Against this must be set the advantage that objects are magnified and with current digital cameras the resolution is very high; areas that would be difficult to inspect in an open procedure are now readily displayed). In laparoscopic dissection the limited range of motion from six to four degrees of freedom can hamper the ability to manipulate instruments and structures (especially for suturing). The necessity to use non-ergonomic instrument positions disrupts the surgeon's hand-eye 
coordination, and the working positions of surgeon and assistant can be awkward. ${ }^{3}$

In certain respects, open surgery retains a clear lead. Procedures performed laparoscopically are generally slower, especially when the setting-up time is included. Laparoscopic nephrectomy, for example, takes 3-4 hours whereas open nephrectomy takes about 1.5 hours. ${ }^{6}$ How many open procedures could be performed in the time lost by a switch to laparoscopic techniques? Also, some procedures are not yet in the repertoire (such as transplant surgery); and in an emergency laparoscopic techniques will often be ruled out by the set-up time, the need to get access quickly and the likelihood that blood will obscure the visual field. Many laparoscopic procedures demand a particular operational conformation (staff and room layout); nevertheless, laparoscopic appendicectomy and duodenal ulcer closure are now standard procedures in many hospitals. Diagnostic laparoscopy has also helped substantially in management of the acute abdomen; and laparoscopic surgery has been found safe and effective in haemodynamically stable patients with abdominal trauma. ${ }^{7}$

The act of initial trocar or needle insertion is not without risk. Vascular and visceral injury (especially to stomach, bowel and bladder) have been reported at $0.25 \%$ and $0.14 \%$, respectively. ${ }^{8}$ In cases of vascular injury, the case fatality rate is between $8.8 \%$ and $13 \%$. The frequency of such injuries is greatest with insertion of the needle and primary trocar since this is done 'blindly'. A lesser complication is trocar site incisional hernia, which develops in about $1 \%$ of patients. ${ }^{9}$ If laparoscopic techniques are deployed for tumour removal, there is a small risk that cancerous cells will seed themselves at the trocar site: such port-site metastasis has been reported after laparoscopic colectomy, ${ }^{10}$ and the risk can be lowered by enclosing the organ in a bag before removal (though this may necessitate enlargement of the incision). ${ }^{3}$ With laparoscopic surgery, inadequate control of bleeding or other difficulties sometimes necessitates conversion to an open procedure, in which case the patient has the worst of both worlds increased time in the operating theatre without the postoperative advantages.

Does the insufflation of $\mathrm{CO}_{2}$ into the abdomen, necessary for the surgeon to identify structures and move the instruments, carry any special hazards? In certain patients at high risk, careful preassessment and monitoring is required to prevent development of acidosis from absorbed $\mathrm{CO}_{2}$. However, in laparoscopic cholecystectomy Zulfikaroglu et al. ${ }^{11}$ report only a negligible decrease in blood $\mathrm{pH}$. Other possible risks arise from the increase in intra-abdominal pressure, resulting in hypertension or arrhythmia, a decrease in venous return from the legs, or even gas embolism.

\section{Training}

All surgeons learn open techniques but experience with laparoscopic techniques is less easily gained - especially when there is pressure to reduce surgical waiting lists. Surgeons with little experience take much longer to complete the procedures and are more likely to make mistakes. This was made clear in a study with a virtualreality trainer: experienced laparoscopic surgeons far outclassed inexperienced surgeons and novices in speed and accuracy. ${ }^{12}$ The practical limitations of laparoscopic surgery are particularly evident with complex operations such as radical prostatectomy. However, once the learning curve has flattened, the results (one-year continence, potency, and positive-margin rates) are comparable with those of retropubic radical prostatectomy. ${ }^{13}$

The acquisition of motor skills can be helped by use of special training devices, ${ }^{14}$ and development of virtual reality and surgical robots (with tremor filtering) proceeds apace. These advantages will improve the performance of trainees in terms of operating time, error and overall efficiency, ${ }^{15-18}$ though their high cost will limit deployment at least in the short term.

\section{SUPPLY AND DEMAND}

Since the history of laparoscopic surgery is short, there have been few long-term comparisons with conventional open operations. This lack of information, however, has not prevented a steady rise in uptake. Market forces are an important influence. For instance, in the USA, the aesthetic benefits of laparoscopic surgery were the reason why many patients insisted on having their gallbladders removed by this method. In the UK, if the Government continues to promote patient choice and autonomy, similar selection pressures could arise in various types of surgery - perhaps resulting in happier patients and enhancing the ethical and medicolegal position of the surgical team. The cost of training and equipment is a limiting factor, but economic analyses need to take account of shorter convalescence times, lower complication rates and earlier return to work.

\section{CONCLUSION}

To return to our original question: does laparoscopic surgery spell the end of the open surgeon? Clearly the answer is no: the skills of open surgery will remain indispensable. But we suspect that, a decade from now, few operations on the abdomen or pelvis will be done by open techniques.

\section{REFERENCES}

1 Cushieri A, Dubois F, Mouiel J, et al. The European experience with laparoscopic cholecystectomy. Am J Surg 1991;161:385-7 
2 Johnston SM, Kidney S, Sweeney KJ, Zaki A, Tanner WA, Keane FV. Changing trends in the management of gallstone disease. Surg Endosc 2003; 17:781-6

3 Rassweiler J, Frede T, Guillonneau B. Advanced laparoscopy. Europ Urol 2002;2:1-12

4 Henry MM, Thompson JN. Clinical Surgery. London: W B Saunders, 2001

5 Risucci DA. Visual spatial perception and surgical competence. Am J Surg 2002;184:291-5

6 Smith D. Renal cancer. Surgery 2002;20:301-4

7 Chol YB, Lim KS. Therapeutic laparoscopy for abdominal trauma. Surg Endosc 2003; 17:421-7

8 Deziel DJ, Millikan KW, Economon SG, Doolas A, Ko ST, Airan MC. Complications of laparoscopic cholecystectomy: a national survey of 4292 hospitals and an analysis of 77604 cases. Am J Surg 1993;165: 9-14

9 Holzinger F, Klaiber C. Trocar site hernias. A rare but potentially dangerous complication of laparoscopic surgery. Chirurg 2002;73: 899-904

10 Savalgi RS, Rosin RD. Port-site metastasis. In: Greene FL, Rosin RD, eds. Minimal Access Surgical Oncology. Abingdon: Radcliffe Medical, 1995:158-65
11 Zulfikaroglu B, Koc M, Soran A, Isman FK, Cinel I. Evaluation of oxidative stress in laparoscopic cholecystectomy. Surg Today 2002;32:869-74

12 Gallagher AG, Satava RM. Virtual reality as a metric for the assessment of laparoscopic psychomotor skills: learning curves and reliability measures. Surg Endosc 2002;16:1746-52

13 Rassweiler J, Sentker L, Seemann O, Hatzinger M, Rumpelt HJ. Laparoscopic radical prostatectomy with the Heilbronn technique: an analysis of the first 180 cases. J Urol 2001;166:2101-8

14 Medina M. The laparoscopic-ring simulation trainer. $J$ Soc Laparoendoscop Surg 2002;6:69-75

15 Hyltander A, Liljegren E, Rhodin PH, Lonroth $\mathrm{H}$. The transfer of basic skills learned in a laparoscopic simulator to the operating room. Surg Endosc 2002;16:1324-8

16 Rosen J, Solazzo M, Hannaford B, Sinanan M. Task decomposition of laparoscopic surgery for objective evaluation of surgical residents' learning curve using hidden Markov model. Computer Aided Surg 2002;7:49-61

17 Seymour NE, Gallagher AG, Roman SA, et al. Virtual reality training improves operating room performance: results of a randomized, double-blinded study. Ann Surg 2002;236:458-63

18 Satava RM. Robotics, telepresence and virtual reality: a critical analysis of the future of surgery. Min Inv Therap 1992;1:357-63 